\title{
Integrated analyses identify the involvement of microRNA-26a in epithelial-mesenchymal transition during idiopathic pulmonary fibrosis
}

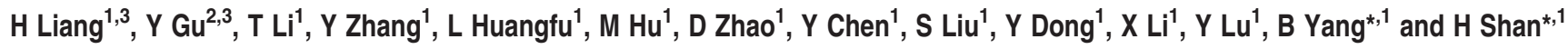

Idiopathic Pulmonary Fibrosis (IPF) is a chronic, progressive, and highly lethal fibrotic lung disease with poor treatment and unknown etiology. Emerging evidence suggests that epithelial-mesenchymal transition (EMT) has an important role in repair and scar formation following epithelial injury during pulmonary fibrosis. Although some miRNAs have been shown to be dysregulated in the pathophysiological processes of IPF, limited studies have payed attention on the participation of miRNAs in EMT in lung fibrosis. In our study, we identified and constructed a regulation network of differentially expressed IPF miRNAs and EMT genes. Additionally, we found the downregulation of miR-26a in mice with experimental pulmonary fibrosis. Further studies showed that miR-26a regulated HMGA2, which is a key factor in the process of EMT and had the maximum number of regulating miRNAs in the regulation network. More importantly, inhibition of miR-26a resulted in lung epithelial cells transforming into myofibroblasts in vitro and in vivo, whereas forced expression of miR-26a alleviated TGF- $\beta 1$ - and BLM-induced EMT in A549 cells and in mice, respectively. Taken together, our study deciphered the essential role of miR-26a in the pathogenesis of EMT in pulmonary fibrosis, and suggests that miR-26a may be a potential therapeutic target for IPF.

Cell Death and Disease (2014) 5, e1238; doi:10.1038/cddis.2014.207; published online 22 May 2014

Subject Category: Experimental Medicine

Idiopathic Pulmonary Fibrosis (IPF) is a chronic, irreversible and lethal lung disorder with unknown etiology. ${ }^{1}$ It has been proposed that IPF likely results from the aberrant activation of alveolar epithelial cells after injury along with the accumulation of inflammation cells, which provoke the excessive proliferation of fibroblasts and exaggerated deposition of extracellular matrix (ECM) in the lung parenchyma, and finally leading to the destruction of lung parenchyma.,3 Although significant progress has been made in recent years in elucidating key aspects of the pathobiology of IPF, the disorder lacks effective treatment strategies. ${ }^{4}$

Although the molecular mechanisms underlying IPF are largely unknown, many scientists believe that the fibroblasts foci formation and excessive ECM production are direct causes of IPF. ${ }^{5,6}$ The key mesenchymal feature of pathological fibrosis is the increased numbers of transdifferentiated fibroblasts, namely, myofibroblasts. Myofibroblasts share features with both fibroblasts and smooth muscle cells; they overexpress a-smooth muscle actin (a-SMA) and are most likely responsible for the enhanced synthesis of abnormal matrix observed in pulmonary fibrosis. ${ }^{7}$ Myofibroblasts have multiple origins in the lung, such as fibroblasts, epithelial cells and bone marrow-derived circulating fibrocytes. Fibroblasts and epithelial cells are the primary origins of myofibroblasts in IPF. ${ }^{8,9}$ According to lineage tracing in murine models of lung fibrosis, it is estimated that up to one-third of fibroblasts may be of epithelial origin. ${ }^{10,11}$ However, the role of epithelialmesenchymal transition (EMT) in lung fibrosis is just beginning to be understood. Therefore, determining the mechanism of EMT involved in the pathogenesis of lung fibrosis is likely to suggest important new directions for the treatment of IPF.

MicroRNAs (miRNAs) are a class of 19-22 nt non-coding small RNAs that control the expression of a large number of genes by binding to the $3^{\prime}$ UTR of targets and blocking translation or by causing degradation of target mRNA. ${ }^{12}$ miRNAs have been implicated in the initiation and progression of pathological processes, including cancer and fibrosis in the heart, liver, kidney and others. ${ }^{13-15}$ Some papers have reported that miRNAs are involved in cancer by regulating the EMT. ${ }^{16,17}$ However, limited studies have payed attention on the participation of miRNAs on EMT in lung fibrosis.

In our study, we focused on investigating the impact and mechanism of miR-26a on EMT involved in IPF and the potential effect on improving treatment for IPF. Our results

\footnotetext{
${ }^{1}$ Department of Pharmacology (State-Province Key Laboratories of Biomedicine-Pharmaceutics of China, Key Laboratory of Cardiovascular Research, Ministry of Education), Harbin Medical University, Harbin, Heilongjiang, People's Republic of China and ${ }^{2}$ College of Bioinformatics Science and Technology, Harbin Medical University, Harbin, Heilongjiang, People's Republic of China

*Corresponding authors: B Yang or H Shan, Department of Pharmacology (State-Province Key Laboratories of Biomedicine-Pharmaceutics of China, Key Laboratory of Cardiovascular Research, Ministry of Education), Harbin Medical University, Xuefu Road 194, Harbin, Heilongjiang 150081, People's Republic of China. Tel: + 86451 86671354; Fax: + 86451 86667511; E-mail: yangbf@ems.hrbmu.edu.cn or shanhongli@ems.hrbmu.edu.cn

${ }^{3}$ These authors contributed equally to this work.

Keywords: microRNA-26a; HMGA2; EMT; Idiopathic Pulmonary Fibrosis

Abbreviations: IPF, idiopathic Pulmonary Fibrosis; ECM, extracellular matrix; a-SMA, a-smooth muscle actin; EMT, epithelial-mesenchymal transition; HMGA2, highmobility group protein A2; NC, negative control; TGF- $\beta 1$, transforming growth factor- $\beta 1$; DAPI, 4',6-diamidino-2-phenylindole; BrdU, 5-Bromo-2-deoxyUridine Received 06.12.13; revised 04.4.14; accepted 08.4.14; Edited by A Stephanou
} 
showed that miR-26a was significantly downregulated in IPF, and the EMT pathway was significantly enriched with the differentially expressed IPF genes. Moreover, inhibition of miR-26a caused apparent EMT in A549 cells and in mice, whereas overexpression of miR-26a retarded Transforming growth factor (TGF)- $\beta 1$ - and bleomycin (BLM)-induced EMT in vitro and in vivo, respectively. These findings indicate that miR-26a is a potential EMT inhibitor and may be considered a new therapeutic tool for IPF.

\section{Results}

EMT pathway is dysregulated in IPF. We detected significantly differentially expressed genes in the mRNA expression profile of IPF compared with the control group and performed functional enrichment using biological process terms in the Gene Ontology database. Our results showed that the EMT pathway (GO:0001837) was significantly enriched with the differentially expressed IPF genes $(P=9.01 \mathrm{E}-4)$. The EMT pathway in the Gene Ontology database contains 79 genes, and 50 of them were detected in the microarray. We found that 35 out of the 50 detected genes were significantly differentially expressed in IPF. Two distinctive expression clusters of 35 EMT pathway genes were obtained by hierarchical clustering analysis (Figure 1). These results demonstrate that the EMT pathway plays important roles in the progression of IPF.

To explore the role of miRNAs on regulating EMT in the process of IPF, we identified the differentially expressed miRNAs from the IPF miRNA expression profile compared with the control group and constructed a regulation network of differentially expressed IPF miRNAs and EMT genes (Figure 2a). The network contains all EMT pathway genes, including the differentially expressed genes, the non-differentially expressed genes and the genes not detected in the mRNA expression profile. We found that high-mobility group protein A2 (HMGA2) was regulated by 33 miRNAs that were differentially expressed in IPF (Figure 2b), which was the maximum number of regulating miRNAs, although HMGA2 was not detected in the mRNA expression profile. This result indicates that HMGA2 may be dysregulated by miRNAs and may participate in the progression of EMT, which further induces IPF. Moreover, miR-26a showed a significant downregulation in the IPF miRNA expression profile $(P=7.17 \mathrm{E}-$ $13)$, and miR-26a regulated many EMT-related genes (Figure 2c), including HMGA2.

miR-26a is downregulated in mice with experimental pulmonary fibrosis. To investigate whether miR-26a participates in the development of pulmonary fibrosis, we constructed a mouse model of pulmonary fibrosis by administering intratracheal BLM, a well-studied pulmonary fibrosis model. ${ }^{18}$ After 4 weeks, there was a significant increase in the deposition and content of collagen in the mice treated with BLM compared with the mice treated with saline (Figures $3 a$ and b). Additionally, the mRNA levels of collagen $1 \alpha 1$ and collagen $3 \alpha 1$ were markedly upregulated in BLM-treated mice (Figure 3c). Real-time PCR analysis found that the expression of miR-26a was significantly decreased in the lungs of mice following administration of BLM (Figure 3d).

It has been reported that TGF- $\beta 1$-induced EMT is the major source of myofibroblasts, which has a substantial role in IPF by secreting ECM components to promote lung fibrosis. In our study, we found that both TGF- $\beta 1$ and $\mathrm{p}$-Smad3 were upregulated at the protein level in the mice receiving BLM. In addition, the expression of HMGA2, a key factor in EMT, was strikingly elevated in the BLM models (Figure $3 e$ ). Furthermore, the expression of E-cadherin was markedly decreased, whereas the expression of $\alpha$-SMA was significantly increased in the lungs of mice with BLM-induced pulmonary fibrosis. These results indicated that EMT occurred in our model of experimental pulmonary fibrosis.

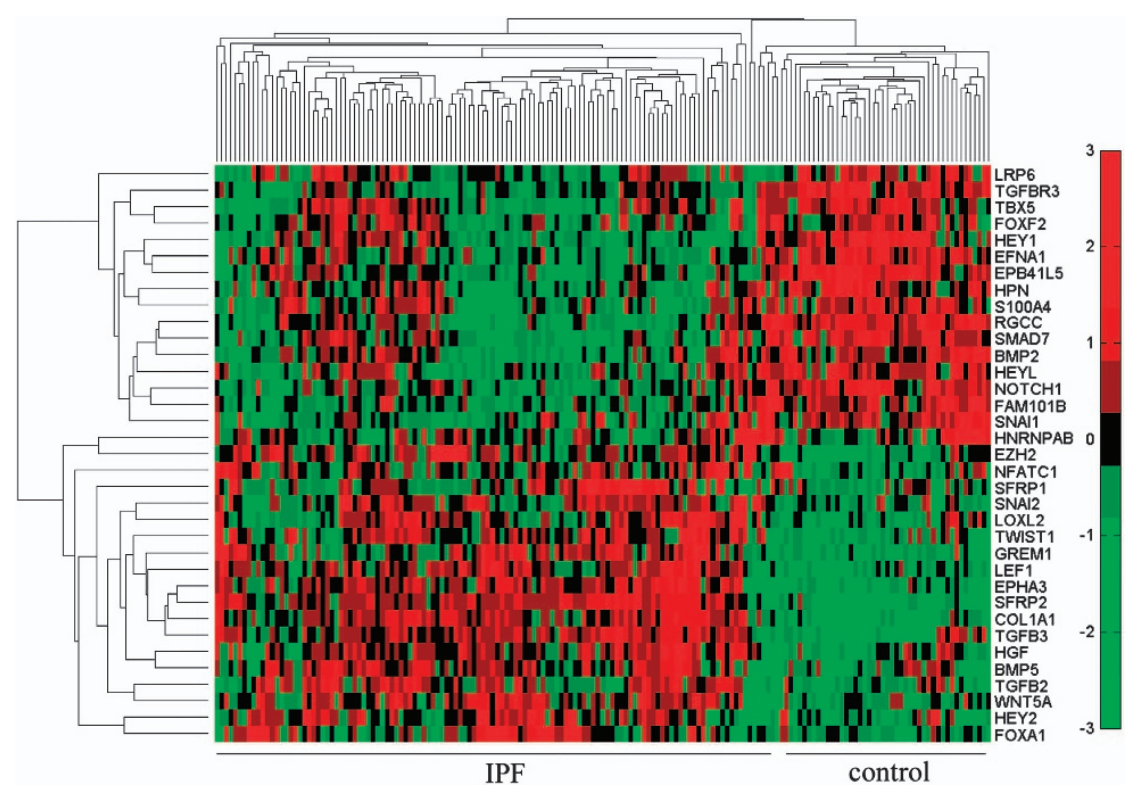

Figure 1 Differentially expressed EMT pathway genes between IPF and control group. Heat map represents the differentially expressed genes (adjusted $P$-value $<0.01$ ) of EMT pathways in IPF group compared with the control group 
a

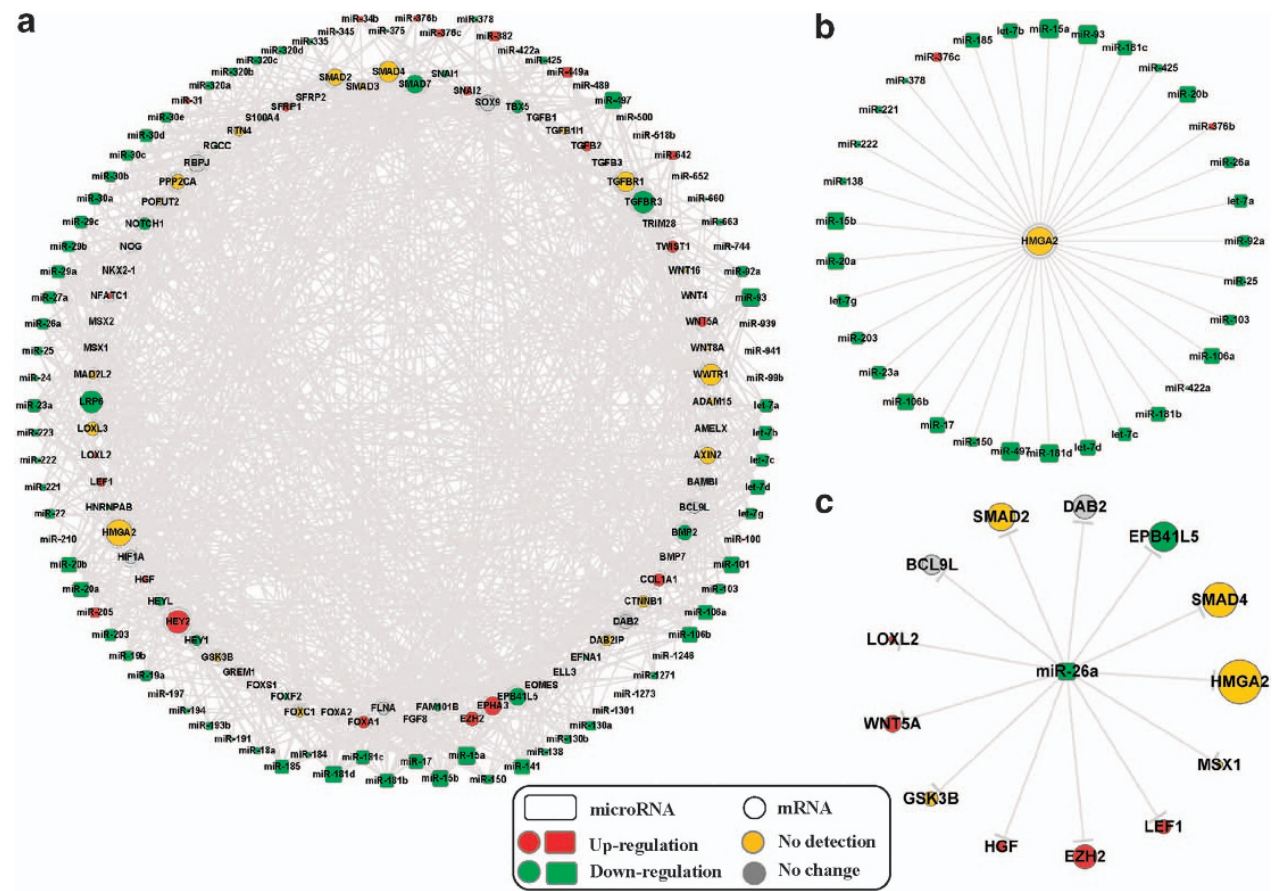

Figure 2 Regulation network of IPF miRNAs and EMT-related genes. (a) The regulation network of differentially expressed miRNA in IPF and EMT pathway genes. (b) The subnetwork of regulation relationship among the miRNAs and HMGA2, which has the maximum number of regulating miRNAs in the regulation network. (c) The network of regulation relationship among miR-26a and its targets. The size of each node corresponds to the number of genes or miRNAs connected to the node

HMGA2 is a direct target of miR-26a in A549 cells. Based on the above findings, we proposed that miR-26a is involved in the process of IPF, and the negative correlation between miR-26a and EMT may participate in the progression of pulmonary fibrosis. Alignment of miR-26a with the HMGA2 $3^{\prime}$-UTR sequence revealed one potential conserved seed site, which appeared in several databases (TargetScan, miRDB, miRanda and PicTar; Figure 4a). As depicted in Figure 4b, transient introduction of miR-26a into A549 cells led to a significant decrease in HMGA2 at the protein level. Moreover, this inhibition was reversed by co-transfection with AMO-26a, and the negative control (NC) had no significant effect. The relationship between miR-26a and HMGA2 was further verified in Figure 4c. Co-transfection of miR-26a with the luciferase reporter carrying a portion of the human HMGA2 3'-UTR caused a significant decrease in luciferase activities compared with transfection with luciferase vector alone. The reduction of luciferase activities was efficiently reversed by AMO-26a $(10 \mathrm{nmol} / \mathrm{l})$. Furthermore, we used an HMGA2 3'-UTR construct that was mutated at the predicted miR-26a site. miR-26a failed to diminish the mutated luciferase activities, whereas the mutated miR-26a was able to do so. Finally, real-time PCR showed that miR-26a had no effect on HMGA2 mRNA levels (Figure 4d) demonstrating that miR-26a directly regulates HMGA2 in a post-transcriptional manner.

Loss of function of miR-26a induces EMT in vitro and in vivo. HMGA2 is a key positive regulator in the process of EMT and induces pulmonary fibrosis in mice. ${ }^{19}$ The direct regulation of miR-26a on HMGA2 demonstrates that the downregulation of miR-26a may contribute to IPF by inducing
EMT. Moreover, miR-26a expression was significantly suppressed by treatment of A549 cells with recombinant TGF- $\beta 1$, a classic EMT model in epithelial cells (Figure 5a). To determine the potential role of miR-26a in the course of pulmonary fibrosis, we transfected AMO-26a into A549 cells. As shown in Figure 5b, AMO-26a effectively inhibited the expression of miR-26a and caused the elevation of collagen content (Figure 5c). Furthermore, the reduction of miR-26a induced an obvious EMT phenotype in A549 cells (Figure $5 d$ ) together with clear alteration of certain related proteins, including E-cadherin, vimentin and $\alpha$-SMA (Figure 5e). Furthermore, we observed apparent EMT and lung fibrosis in the mice treated with an intratracheal injection of antagomiR-26a (Figure 6), which were observations similar to those detected in vitro. These results suggest that loss of function of miR-26a could facilitate lung epithelial cells to transform into myofibroblasts and induce pulmonary fibrosis in mice.

Forced expression of miR-26a diminishes EMT and fibrogenesis in A549 cells and in mice with experimental pulmonary fibrosis. To evaluate the effects of miR-26a on EMT and lung fibrosis, we examined whether forced expression of miR-26a could mitigate the phenotype of EMT induced by TGF- $\beta 1$ in A549 cells. We transfected miR26 a into cultured A549 cells to assess the effects of miR-26a on collagen production. As shown in Figure 7a, miR-26a abolished TGF- $\beta 1$-induced secretion of collagen in A549 cells, while the NC group failed to do so. Additionally, immunofluorescence and Western blot assays showed that miR-26a attenuated TGF- $\beta 1$-induced EMT in A549 cells (Figures $7 b$ and $c$ ). 


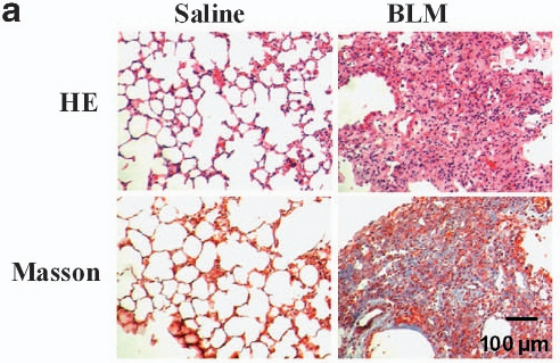

b
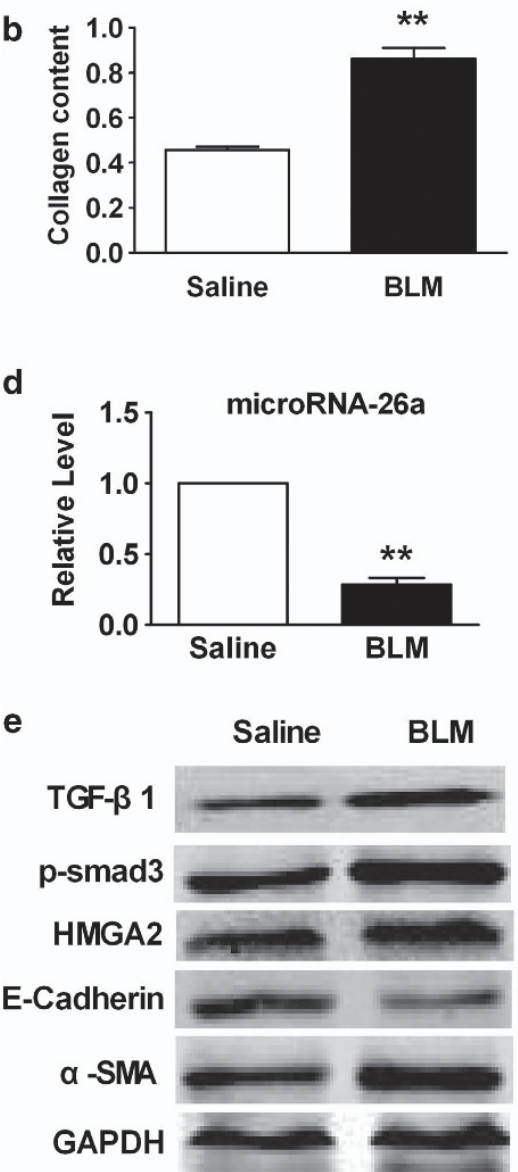
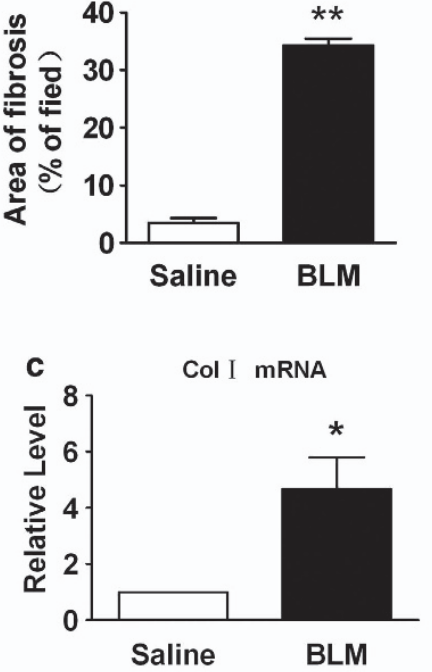

Col III mRNA

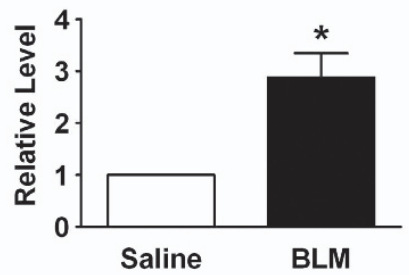

$12 \mathrm{kDa}$

$52 \mathrm{kDa}$

$18 \mathrm{kDa}$

120 kDa

$40 \mathrm{kDa}$

$37 \mathrm{kDa}$

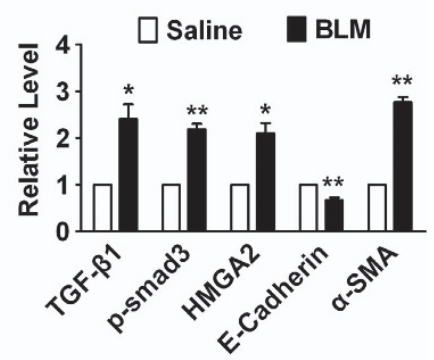

Figure 3 miR-26a is downregulated in lungs from BLM-treated mice. (a) HE and Masson Trichrome staining of mouse lung sections shows interstitial fibrosis with collagen deposition 28 days after injection of BLM (magnification, $200 \times$ ). (b) Increased collagen content in the lungs of mice treated with BLM compared with saline group. (c) Increased collagen I and collagen III mRNA expression in the BLM-treated mice compared with control animals, measured by real-time PCR. (d) Real-time PCR analysis of decreased expression of miR-26a in the lungs of mice with experimental pulmonary fibrosis. (e) Western blot analysis of proteins in the TGF- $\beta$ signaling pathway and EMT-related genes in BLM-treated mice as compared with control animals. Mean \pm S.E.M.; $n=5$ mice in each group; ${ }^{*} P<0.05,{ }^{* *} P<0.01$ versus saline group

To examine whether miR-26a has protective roles in the progression of lung fibrosis, we administrated an intratracheal injection of agomiR-26a 7 days after instillation of BLM and analyzed the extent of EMT and fibrosis 28 days after administration of BLM. As shown in Figures $7 \mathrm{~d}-\mathrm{f}$, agomiR26 a significantly suppressed collagen content as well as the occurrence of EMT in BLM-treated lungs.

\section{Discussion}

In the present study, we took bioinformatic methods to identify miRNAs potentially involved in EMT under condition of pulmonary fibrosis. Combined with BLM-induced model of pulmonary fibrosis in mice, we confirmed the downregulation of miR-26a in fibrotic lungs. This reduction correlated with increased expression of mesenchymal cells and EMT-related genes. Further studies showed that HMGA2, a key positive regulatory factor in EMT, is one of the direct targets of miR26a. Moreover, miR-26a could be suppressed by TGF- $\beta 1$ in A549 cells, and knockdown of miR-26a caused EMT in vitro and in vivo. Interestingly, forced expression of miR-26a diminished EMT and fibrogenesis in A549 cells and in mice with experimental pulmonary fibrosis. Taken together, our study supports a role for miR-26a in the pathogenesis of EMT 

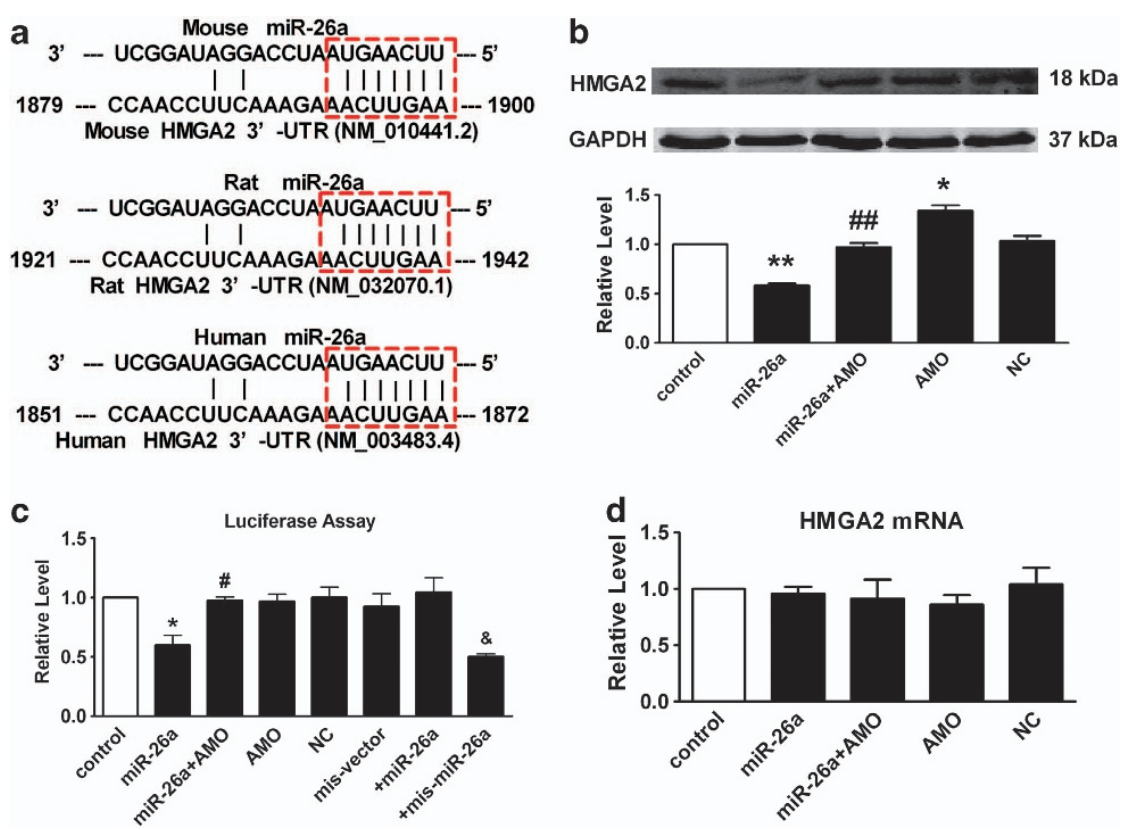

Figure 4 miR-26a post-transcriptionally regulates HMGA2. (a) Sequence alignment showing miR-26a/HMGA2 complementarity for mouse, rat and human genes. The matched base pairs are outlined by dashed red rectangles. The Genbank accession numbers of the genes are indicated in the brackets, and the positions of the target sites are numbered. (b) Compared with control, transfection of miR-26a results in a significant decrease of HMGA2. Co-application of miR-26a with AMO-26a alleviated the reduction of HMGA2, whereas NC showed no effects. (c) Compared with control, transfection of miR-26a with the luciferase reporter gene vector containing the wild-type $3^{\prime}$ UTR of HMGA2 results in a significant decrease of luciferase activity. Co-application of miR-26a with AMO-26a alleviated the reduction of luciferase activity, whereas NC showed no effects. (d) Real-time PCR shows that miR-26a had no effects on HMGA2 mRNA level. AMO/miR-26a inhibitor, AMO-26a; NC/negative control. Mean \pm S.E.M.; $n=4$ represents three independent experiment under each condition; ${ }^{*} P<0.05$, ${ }^{\star *} P<0.01$ versus control; ${ }^{\#} P<0.05,{ }^{\# \#} P<0.01$ versus miR-26a, ${ }^{\&} P<0.05$ versus mis-vector

in pulmonary fibrosis, and suggests that miR-26a may be a potential therapeutic target for IPF.

TGF- $\beta 1$ has been shown to have key roles in pulmonary fibrosis, not only through stimulating proliferation of fibroblasts, but also through induction of EMT in alveolar epithelial cells by activating Smad- or non-Smad signaling pathways. ${ }^{20}$ The EMT process is characterized by the loss of epithelial cell phenotypes, such as diminished expression of E-cadherin and ZO-1, and the acquisition of mesenchymal cell phenotypes, such as enhanced expression of vimentin, $\alpha$-SMA and $\mathrm{N}$-cadherin. EMT converts adherent epithelial cells to motile mesenchymal cells with enhanced migratory capacity, invasiveness, elevated resistance to apoptosis and greatly increased production of ECM components. ${ }^{8}$ Emerging evidence suggests that EMT participates in embryonic development, tissue repair and pathological processes, notably tumor invasiveness and metastasis. ${ }^{21}$

Although large number of studies have confirmed the mechanism of EMT in different cancer types, little is known regarding the role of EMT in IPF. Current studies show that the role of EMT in the formation of activated myofibroblasts during the process of pulmonary fibrosis is still controversial. ${ }^{22,23}$ Yamada et al. ${ }^{24}$ did not find histological evidence for EMT in tissue samples from patients diagnosed with IPF and nonspecific interstitial pneumonia using dual immunohistochemistry. Roch et al. ${ }^{25}$ did not find evidence at the cellular or molecular level for the EMT of labeled cells into myofibroblasts by analyzing normal and fibrotic mouse and human lungs. On the contrary, an increasing number of studies have identified a major role of EMT in lung fibroblasts accumulation in IPF. ${ }^{26,27} \mathrm{~A}$ recent study by Chen $\mathrm{YL}$ et al. ${ }^{28}$ reported that sorafenib ameliorates BLM-induced pulmonary fibrosis along with the inhibition of EMT and fibroblast activation. Sonnylal S et al. $^{29}$ found that overexpression of connective tissue growth factor (CTGF) in epithelial cells causes EMT-like morphological changes and expression of $\alpha-S M A$, which in turn contribute to lung fibrosis. Balli $D$ et al. found that foxm 1 is required for radiation-induced pulmonary fibrosis by enhancing expression of the genes critical for lung inflammation and EMT. ${ }^{30}$ Our results are consistent with these abovementioned findings that the EMT is likely an important mechanism for IPF. Our results presented here strongly suggest for the first time the role of EMT in IPF by large-scale approach in numerous IPF samples. Additionally, the regulation network constructed by the differentially expressed miRNAs and EMTrelated genes in IPF should also aid better understanding of the mechanisms for IPF.

To examine the role of miRNAs on EMT, many scientists have studied the effects of miRNAs on the direct transcription factors related to EMT. Chang et al. ${ }^{31}$ found that miR-200c has a role in regulating both EMT and EMT-associated stem cell properties in breast cancer through directly regulating ZEB1. Yang et al. ${ }^{32}$ confirmed that miR-506 is a potent EMT inhibitor and therapeutic tool in serious ovarian cancer by targeting Snail2. Previous studies have confirmed that HMGA2 is a critical factor required for TGF- $\beta 1$-induced EMT in mammary epithelial cells. ${ }^{33}$ In mammary epithelial cells, TGF- $\beta 1$, via the Smad pathway, induces the expression of HMGA2, which binds to the Snail1 promoter and induces Snail1 expression, E-cadherin repression and the overall EMT 

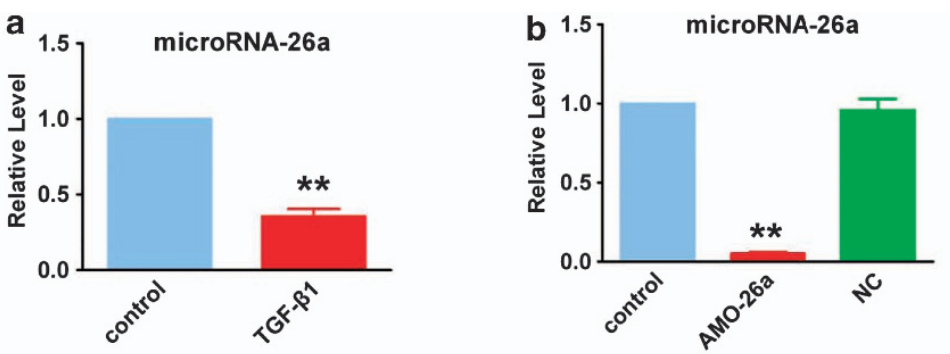

d
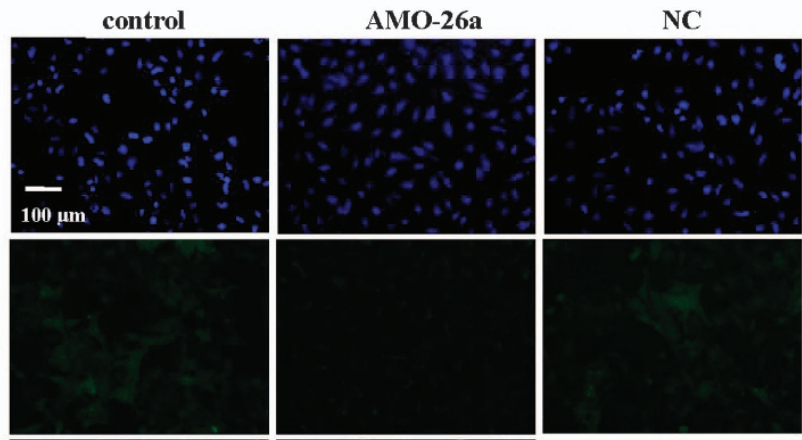

E-cadherin
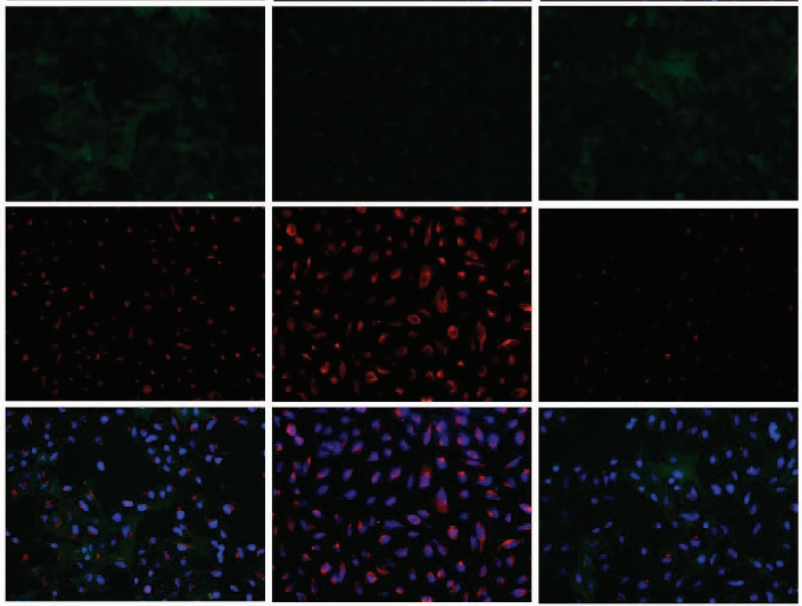

c
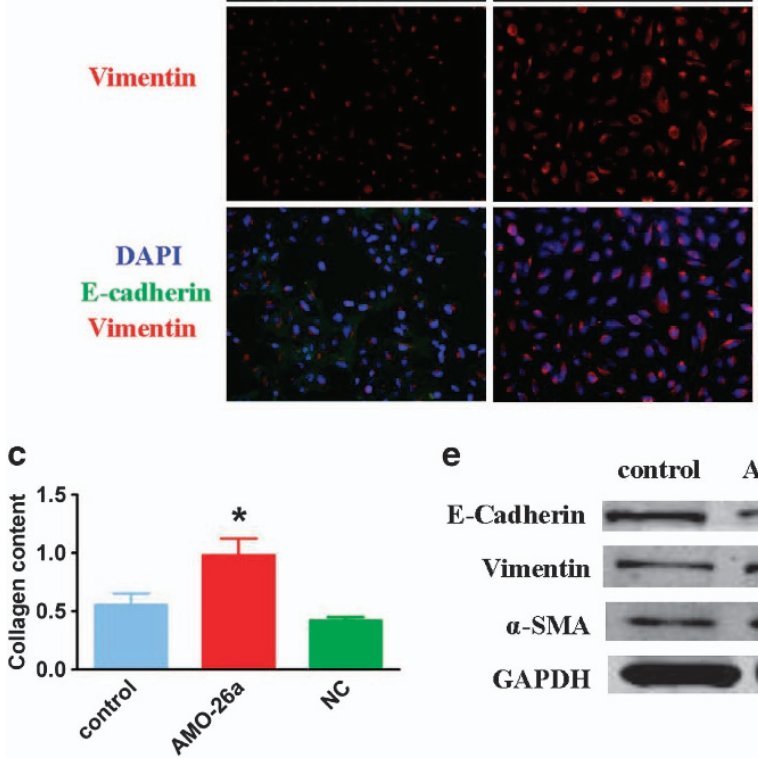

e

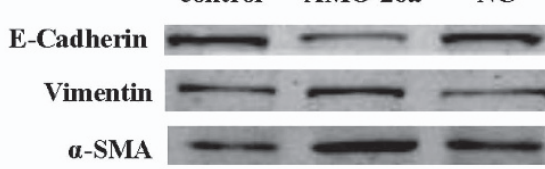

$120 \mathrm{kDa}$

$57 \mathrm{kDa}$

(2)

$40 \mathrm{kDa}$

GAPDH

$37 \mathrm{kDa}$

Figure 5 Inhibition of miR-26a causes EMT in cultured A549 cells. (a) TGF- $\beta 1$ inhibits the expression of miR-26a in cultured A549 cells. (b) Real-time PCR analysis of miR-26a expression in A549 cells transfection of AMO-26a as compared with control. (c) Increased collagen content in A549 cells transfected with AMO-26a. Immunofluorescence (d) and Western blot (e) examine the effect of AMO-26a on EMT. $n=4 ;{ }^{*} P<0.05,{ }^{* \star} P<0.01$ versus control

phenotype. $^{34,35}$ However, few studies have focused on the role of HMGA2 in the process of IPF. In our study, we found that HMGA2 had the maximum number of regulating miRNAs in IPF-related miRNA-EMT gene regulation network. Therefore, we supposed that influencing HMGA2 instead of its downstream effectors may be a more effective way to control the process of EMT. Our study revealed that HMGA2 upregulation by miR-26a inhibition is a new mechanism for the EMT involved in pulmonary fibrosis.

The screening and study of miRNA involvement in pulmonary fibrosis is still in the early stages. Two recently published studies have confirmed the effect of dysregulated expression of let-7d and miR-200 on EMT in pulmonary fibrosis. ${ }^{3,36}$ Pandit KV et al. found that let-7d was significantly reduced in human IPF tissues, and the inhibition of let-7 in epithelial cell lines in vitro and in vivo resulted in increased expression of mesenchymal-specific genes and pulmonary fibrosis. ${ }^{3}$ Studies from Yang et al. ${ }^{36}$ confirmed the downregulation of the miR-200 family in the lungs of mice with experimental lung fibrosis and patients with IPF.

Accumulating evidence has confirmed that miR-26a is involved in the pathological processes of many diseases, such as cardiac fibrosis, atrial fibrillation, angiogenesis and others. ${ }^{37-39}$ However, the role of miR-26a on IPF, especially on EMT, remains largely unknown. In this study, we found dysregulated expression of miR-26a in IPF. In addition, we showed that miR-26a could regulate the expression of HMGA2 and attenuate EMT, which suggests 

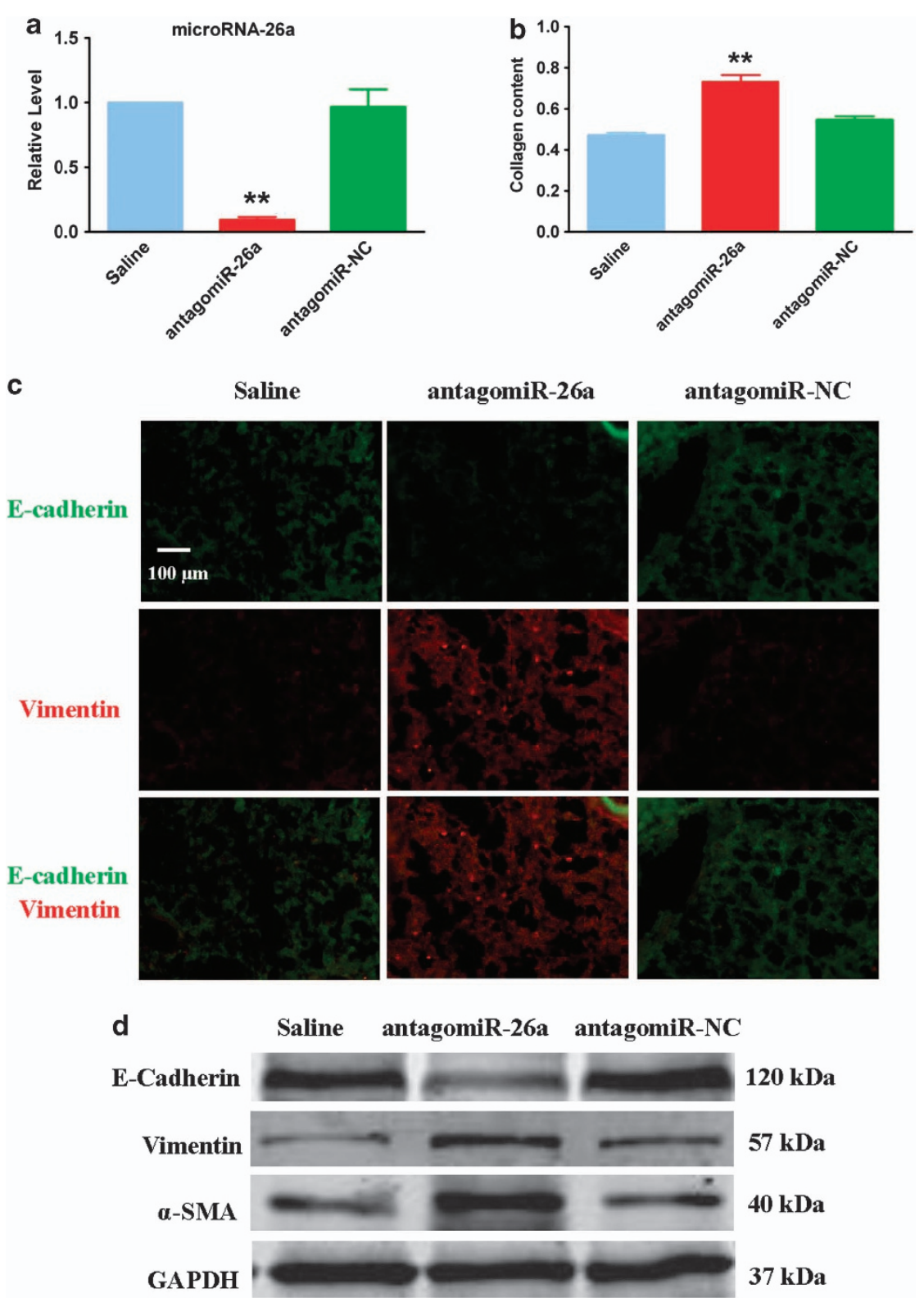

Figure 6 Inhibition of miR-26a in the lung promotes EMT in mice. (a) Real-time PCR analysis of miR-26a expression in mice treated with antagomiR-26a compared with the saline group. (b) Increased collagen content in mice treated with antagomiR-26a. Immunofluorescence (c) and Western blot (d) assays were used to assess the EMT phenotype in antagomiR-26a-treated mice compared with control animals. $n=4 ;{ }^{* *} P<0.01$ versus saline

that miR-26a could serve as a mediator to prevent fibrotic lung diseases.

Numerous studies have indicated that miR-26a has a protective role in the process of cardiac fibrosis by regulating the activation of cardiac fibroblasts. ${ }^{40,41}$ Therefore, we assume that miR-26a also has an important role in modulating the lung fibroblast phenotype based on the following: (1) Similar physiological characteristics and pro-fibrotic pathways exist in lung and cardiac fibroblasts. (2) Our study found that HMGA2 was a direct target of miR-26a in A549 cells. Huleihel L et al. ${ }^{42}$ confirmed that let-7d significantly attenuated the differentiation of fibroblasts into pathological myofibroblasts by targeting HMGA2. (3) The data obtained from databases (TargetScan, miRDB, miRanda and PicTar) showed that miR-26a has many potential targets involved in the regulation of fibroblast phenotype and function, such as CTGF, fibroblast growth factor receptor, hepatocyte growth factor and so forth. IPF is a complex pathological process characterized by excessive fibroblast proliferation and injury of alveolar epithelial cells. Hence, it is undeniable that the anti-fibrotic effect of miR-26a in IPF is a combined effect mediated by a variety of mechanisms. Further studies are needed to reveal other potential mechanisms involved in the anti-fibrotic effects of miR-26a and assess the combined effects of miR-26a in IPF.

In addition to revealing the role and mechanism of miR-26a in EMT, our study explored the potential therapeutic value of miR-26a for IPF. Although many miRNAs have been shown to have considerable roles in regulating numerous diseases, the 
a

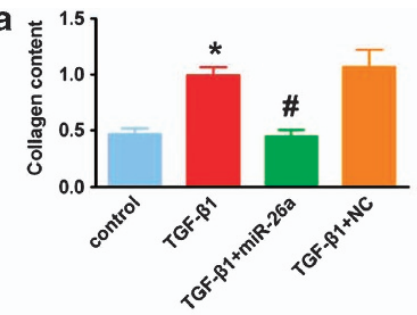

C

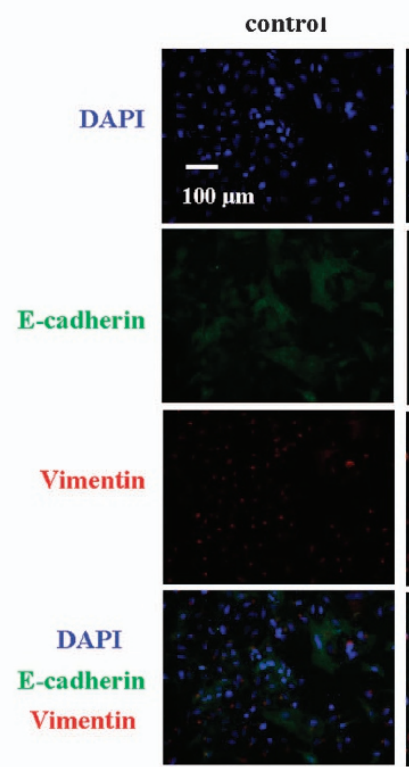

b

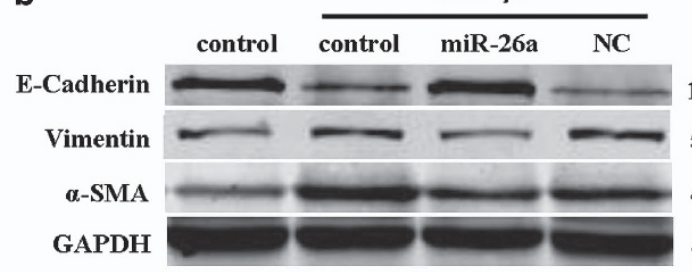

$120 \mathrm{kDa}$

$57 \mathrm{kDa}$

$40 \mathrm{kDa}$

37 kDa
TGF- $\beta 1$

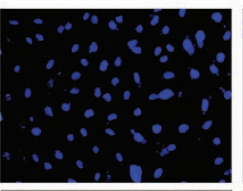

TGF- $\beta 1+m i R-26 a$
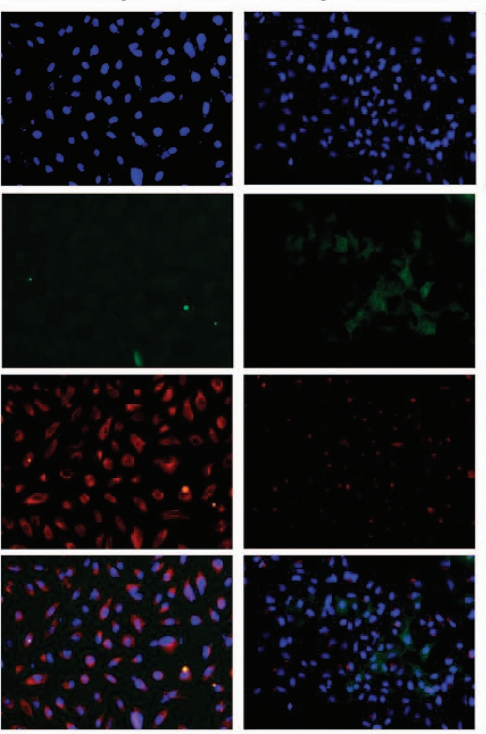

TGF- $\beta 1+$ NC
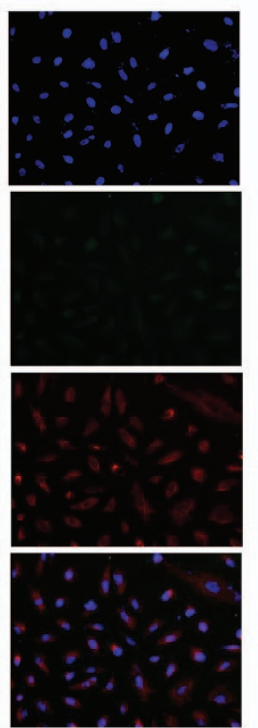

e

BLM
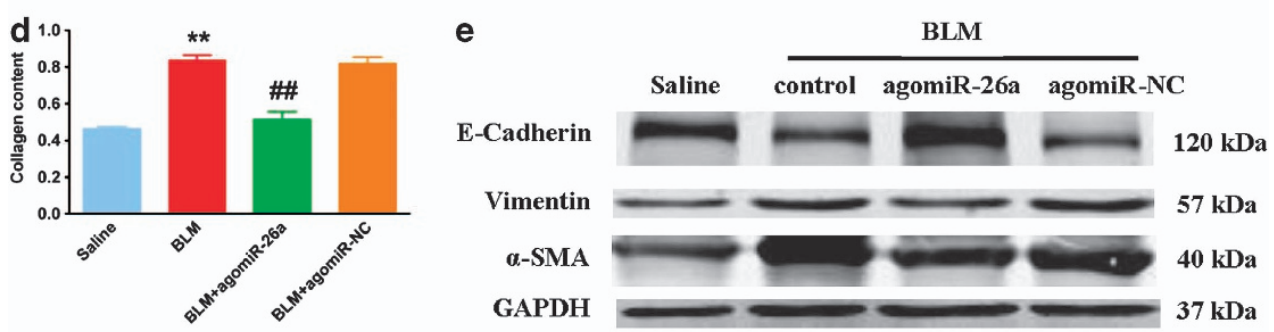

f

Saline

BLM BLM+agomiR-26a BLM+agomiR-NC
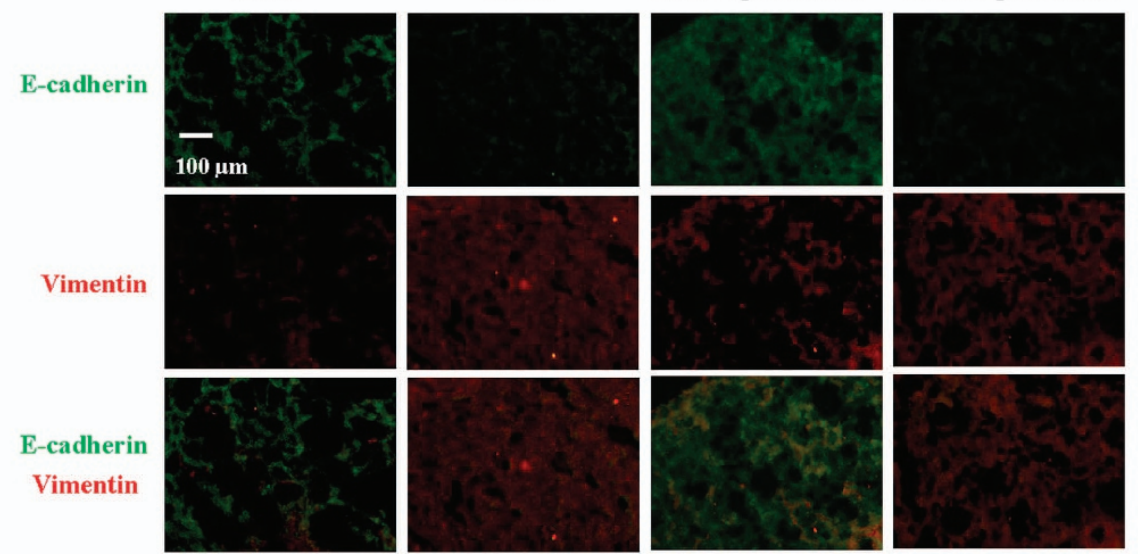

Figure 7 Enhancement of miR-26a expression attenuates EMT in vitro and in vivo. (a) miR-26a suppresses the TGF- $\beta 1$-induced increase in collagen content in A549 cells. Western blot (b) and immunofluorescence (c) assays found that miR-26a suppresses TGF- $\beta 1$-induced EMT in A549 cells. (d) Administration of agomiR-26a attenuates collagen content in bleomycin-treated mice, whereas agomiR-NC has no effect. Western blot (e) and immunofluorescence (f) assays shows that agomiR-26a attenuates EMT in the lungs of mice with pulmonary fibrosis. E-cadherin is stained in green, Vimentin is in red and the nuclei are in blue (magnification, $200 \times)$. $n=4$; ${ }^{\star} P<0.05$ versus control, $\# P<0.05$ versus TGF- $\beta 1 ;{ }^{* \star} P<0.01$ versus saline, ${ }^{\# \#} P<0.01$ versus BLM 
complexity of delivering miRNA in vivo and pharmacokinetics have limited their application in human study. Future studies are needed to validate the clinical value of miR-26a for IPF treatment.

\begin{abstract}
Materials and Methods
Microarray datasets and detection of differentially expressed miRNAs and genes. The microarray datasets were downloaded from the database of Gene Expression Omnibus (http://www.ncbi.nlm.nih.gov/geo/). The mRNA expression profile consists of 119 IPF patient samples and 50 nondiseased control samples (GSE32537). The miRNA expression profile has 106 IPF patient samples and 50 non-diseased control samples (GSE32538). ${ }^{43}$ The quantile normalization and log2 transformation were applied to the raw data. The values of replicate probes corresponding to a miRNA or a gene were averaged to represent the expression value of the miRNA or gene. A two-sample t-test was performed to detect differentially expressed miRNAs and genes, respectively. The $P$ values were adjusted by the Benjamini and Hochberg $(\mathrm{BH})$ correction procedure to account for multiple tests with false discovery rate $<1 \%$.
\end{abstract}

miRNA targets databases and pathway information. Considering that miRNA targets predicted by multiple algorithms might be more reliable, we used the miRNA-target interactions of Homo sapiens appearing in at least two of the nine datasets (TargetScan, miRanda, PicTar, miRBase, DIANA-microT, PITA, RNAhybrid, miRTarBase and miRecords). The human genes annotated in the biological process of 'EMT' were downloaded from the Gene Ontology database (GO:0001837). The hypergeometric distribution model was used to test whether the EMT pathway was enriched with differentially expressed IPF genes. Hierarchical clustering of expression values was performed with $\mathrm{R}$ software using the metric of Euclidean distance and average linkage.

Experimental pulmonary fibrosis model and treatment. Animals (8-week-old C57BL/6 mice; 20-30 g) in this work were used in accordance with the regulations of the Ethic Committees of the Harbin Medical University and conformed to the Guide for the Care and Use of Laboratory Animals published by the US National Institutes of Health (NIH Publication No. 85-23, revised 1996). The mice were anesthetized with pentobarbital $(40 \mathrm{mg} / \mathrm{kg}$, i.v.) and randomly divided into two groups: saline and BLM. To induce pulmonary fibrosis, BLM was intratracheally injected at a dose of $1.5 \mathrm{U} / \mathrm{kg}$ body weight on Day 0 , and the mice were killed at 4 weeks after the BLM treatment. The control group received the same volume of sterile saline. All surgical procedures were performed under sterile conditions. The cholesterol-conjugated miR-26a mimics/antisense and NC (agomiR-26a/antagomiR-26a and agomir-NC/antagomiR-NC, respectively) were purchased from RiboBio Co., Ltd. (Guangzhou, China). Mice were treated either with agomiR-26a/antagomiR-26a (10 mg/kg body weight in $50 \mu \mathrm{l}$ saline) or agomiR-NC/antagomiR-NC by intratracheal injection.

Masson's trichrome staining. The lungs of mice in the BLM and saline groups were quickly dissected and immersed in $4 \%$ neutral buffered formalin for $24 \mathrm{~h}$ and stained with Masson's trichrome. Fibrosis tissue was quantified with Image Pro Plus 6.0 (MediCybernetics, Silver Spring, MA, USA). Three areas were analyzed in each slide, and each area was divided into 100 squares. The number of collagen points (blue stain) was scored as 1 (present) or 0 (absent). The results are shown as the percentage of area occupied by fibrosis to the total area.

Collagen content assay. Total collagen content was measured using the Sircol Collagen Assay Kit (Biocolor, Belfast, Northern Ireland) according to the manufacturer's instruction. Each sample was incubated at $4{ }^{\circ} \mathrm{C}$ with stirring for $24 \mathrm{~h}$. After centrifugation, $100 \mu \mathrm{l}$ of each supernatant was assayed. One milliliter of Sircol dye reagent bound to collagen was added to each sample, and the solution was mixed at $4{ }^{\circ} \mathrm{C}$ for $30 \mathrm{~min}$. After centrifugation, the pellet was suspended in $700 \mu \mathrm{l}$ of the alkali reagent included in the kit and read at $540 \mathrm{~nm}$ with a spectrophotometer. Collagen standard solution was utilized to construct a standard curve. Total collagen content was determined by assaying cell protein content. The amount of protein was colorimetrically assessed at $562 \mathrm{~nm}$ with a BCA protein assay kit (Beyotime, Nantong, China).

Real-time RT-PCR. Total RNA samples were extracted from the lung tissues of mice or A549 cells using Trizol (Invitrogen, Carlsbad, CA, USA). As delineated in the work of Yang et al., ${ }^{44}$ the relative expression levels of mRNAs and miRNAs were quantified by the mirVana qRT-PCR miRNA Detection Kit (Applied Biosystems, Foster City, CA, USA), and real-time RT-PCR with TaqMan microRNA assays (Applied Biosystems), respectively. After the cycle reactions, the threshold cycle (Ct) was determined and relative mRNA and miRNA levels were calculated based on the $\mathrm{Ct}$ values and normalized to GAPDH or U6 levels in each sample.

Cell culture and treatment. A549 cells were purchased from the Cell Bank of Chinese Academy of Sciences (Shanghai, China). The cells were grown in 25$\mathrm{cm}^{2}$ cell culture flasks with F-12K medium (Hyclone, Logan, UT, USA) containing $10 \%$ fetal bovine serum (Hyclone), $2 \mathrm{mM} \mathrm{L}$-glutamate, $100 \mathrm{U} / \mathrm{ml}$ penicillin $\mathrm{G}$, and $100 \mathrm{U} / \mathrm{ml}$ streptomycin at $37^{\circ} \mathrm{C}$ in $5 \% \mathrm{CO}_{2}, 95 \%$ air. The cells were maintained at sub-confluent densities and used for experiments when reaching passages 3 or 4 . After starvation in serum-free medium for $24 \mathrm{~h}$, A549 cells were administered recombinant human TGF- $\beta 1$ (Sigma-Aldrich, St. Louis, MO, USA) for $48 \mathrm{~h}$.

Transfection procedures. For transfections, the cells were washed with serum-free medium once and then incubated with $4 \mathrm{ml}$ of serum-free medium for 4-6 $\mathrm{h}$. The miRNA and Lipofectamine 2000 (Invitrogen) were separately mixed with $500 \mu \mathrm{l}$ of Opti-MEM I Reduced Serum Medium (Gibco, Grand Island, NY, USA) for $5 \mathrm{~min}$. Then, the two mixtures were combined and incubated at room temperature for $20 \mathrm{~min}$. The Lipofectamine-miRNA mixture was added to the cells and incubated at $37^{\circ} \mathrm{C}$ for $6 \mathrm{~h}$. Subsequently, $4 \mathrm{ml}$ of fresh medium containing $10 \%$ FBS was added to the flasks and the cells were maintained in the culture until the following experiments.

Immunofluorescence staining. Cultured A549 cells on sterile glass cover slips were briefly washed with cold PBS 3 times and fixed with 4\% paraformaldehyde for $15 \mathrm{~min}$. Then, the cell membrane was penetrated by Triton $\mathrm{X}-100$ for $1 \mathrm{~h}$ and blocked by normal goat serum for $1 \mathrm{~h}$ at $37^{\circ} \mathrm{C}$. The cells were incubated with anti-E-cadherin or anti-vimentin antibody (Abcam Inc., Cambridge, MA, USA; Cell Signaling Technology, Beverly, MA, USA) overnight at $4{ }^{\circ} \mathrm{C}$ and subsequently incubated with FITC-conjugated goat anti-mouse or goat anti-rabbit antibody for $1 \mathrm{~h}$. The cells were washed with PBS, and the nuclei were stained with DAPI (Roche Molecular Biochemicals, Basel, Switzerland) for $5 \mathrm{~min}$ at room temperature. Immunofluorescence was analyzed under a fluorescence microscope (Nikon $80 \mathrm{i}$, Japan).

Western blotting. For Western blot analysis, total protein samples were extracted from tissues or cells with the procedure as previously described. ${ }^{45}$ Tissues or cells were lysed with RIPA lysis buffer (Beyotime). A total of $50 \mu \mathrm{g}$ of protein was fractionated on a $10 \%$ or $15 \%$ SDS-polyacrylamide gel. After electrophoretically transferring to a Pure Nitrocellulose Blotting membrane (Pall Life Sciences, Ann Arbor, MI, USA), the blots were probed with primary antibodies, with GAPDH (antiGAPDH antibody from Kangchen, Shanghai, China) as an internal control. Primary antibodies against TGF- $\beta 1, \mathrm{p}$-Smad3 and vimentin were rabbit polyclonal antibodies that were purchased from Cell Signaling Technology. Primary antibodies against E-cadherin or $\alpha$-SMA were mouse polyclonal antibodies that were purchased from Abcam Inc. The immunoreactivity was detected using Odyssey Infrared Imaging System (Gene Company Limited, Hong Kong, China). The bands were quantified by measuring the band intensity for each group.

Luciferase reporter assays. HMGA2 $3^{\prime}$-UTR containing the conserved miR26a binding sites was synthesized by Invitrogen and amplified by PCR. The PCR fragment was subcloned into the Sacl and Hindlll sites downstream the luciferase gene in the pMIR-REPORT Luciferase vector (Applied Biosystems). The luciferase vector (100 ng) containing the $3^{\prime}$ UTR was cotransfected with miR-26a mimics or AMO-26a into HEK-293 cells using Lipofectamine 2000 (Invitrogen). As an internal control, $10 \mathrm{ng}$ of Renilla luciferase reporters were also included. After a 36-h transfection, the cells were collected, and the dual luciferase activities were measured using a luminometer (Promega, Madison, WI, USA) according to the manufacturer's instructions.

Statistical analyses. All data are presented as the mean \pm S.E.M. One way analysis of variance ANOVA followed by Dunnett's test was used for multiple comparisons. A two-tailed value of $P<0.05$ was considered a significant difference. The data were analyzed using GraphPad Prism 5.0 (GraphPad Inc, San Diego, CA, USA) and SPSS 14.0 (SPSS Inc, Chicago, IL, USA). 


\section{Conflict of Interest}

The authors declare no conflict of interest.

Acknowledgements. This study was supported by the National Basic Research Program of China (973 program, 2013CB531104); the Funds for Creative Research Groups of the National Natural Science Foundation of China grant (81121003); the National Natural Science Foundation of China (81201822; 31300943); Research Fund for the Doctoral Program of Higher Education of China (20132307110004).

1. Selman M, Pardo A, Kaminski N. Idiopathic pulmonary fibrosis: aberrant recapitulation of developmental programs? PLoS Med 2008; 5: e62.

2. Liu G, Friggeri A, Yang Y, Milosevic J, Ding Q, Thannickal VJ et al. miR-21 mediates fibrogenic activation of pulmonary fibroblasts and lung fibrosis. $J$ Exp Med 2010; 207: 1589-1597.

3. Pandit KV, Corcoran D, Yousef H, Yarlagadda M, Tzouvelekis A, Gibson KF et al. Inhibition and role of let-7d in idiopathic pulmonary fibrosis. Am J Respir Crit Care Med 2010; 182: 220-229.

4. Selman M, Pardo A, Richeldi L, Cerri S. Emerging drugs for idiopathic pulmonary fibrosis. Expert Opin Emerg Drugs 2011; 16: 341-362.

5. Hung C, Linn G, Chow YH, Kobayashi A, Mittelsteadt K, Altemeier WA et al. Role of lung pericytes and resident fibroblasts in the pathogenesis of pulmonary fibrosis. Am J Respir Crit Care Med 2013; 188: 820-830.

6. Phan SH. Genesis of the myofibroblast in lung injury and fibrosis. Proc Am Thorac Soc 2012; 9: 148-152.

7. Scotton CJ, Chambers RC. Molecular targets in pulmonary fibrosis: the myofibroblast in focus. Chest 2007; 132: 1311-1321.

8. Kalluri R, Weinberg RA. The basics of epithelial-mesenchymal transition. J Clin Invest 2009; 119: 1420-1428.

9. Hinz B, Phan SH, Thannickal VJ, Galli A, Bochaton-Piallat ML, Gabbiani G. The myofibroblast: one function, multiple origins. Am J Pathol 2007; 170: 1807-1816.

10. Tanjore H, Xu XC, Polosukhin VV, Degryse AL, Li B, Han W et al. Contribution of epithelialderived fibroblasts to bleomycin-induced lung fibrosis. Am J Respir Crit Care Med 2009; 180: 657-665.

11. Kim KK, Kugler MC, Wolters PJ, Robillard L, Galvez MG, Brumwell AN et al. Alveolar epithelial cell mesenchymal transition develops in vivo during pulmonary fibrosis and is regulated by the extracellular matrix. Proc Natl Acad Sci USA 2006; 103: 13180-13185.

12. Inui M, Martello G, Piccolo S. MicroRNA control of signal transduction. Nat Rev Mol Cell Biol 2010; 11: 252-263.

13. Chou J, Shahi P, Werb Z. microRNA-mediated regulation of the tumor microenvironment. Cell Cycle 2013; 12: 20.

14. Bowen T, Jenkins RH, Fraser DJ. MicroRNAs, transforming growth factor beta-1, and tissue fibrosis. J Pathol 2013; 229: 274-285.

15. Pan Z, Sun X, Shan H, Wang N, Wang J, Ren J et al. MicroRNA-101 inhibited postinfarct cardiac fibrosis and improved left ventricular compliance via the FBJ osteosarcoma oncogene/transforming growth factor-beta1 pathway. Circulation 2012; 126: 840-850.

16. Taube JH, Malouf GG, Lu E, Sphyris N, Vijay V, Ramachandran PP et al. Epigenetic silencing of microRNA-203 is required for EMT and cancer stem cell properties. Sci Rep 2013; 3: 2687

17. Zheng H, Li W, Wang Y, Xie T, Cai Y, Wang Z et al. miR-23a inhibits E-cadherin expression and is regulated by AP-1 and NFAT4 complex during Fas-induced EMT in gastrointestinal cancer. Carcinogenesis 2014; 35: 173-183.

18. Moore BB, Hogaboam CM. Murine models of pulmonary fibrosis. Am J Physiol Lung Cell Mol Physiol 2008; 294: L152-L160.

19. Willis BC, Borok Z. TGF-beta-induced EMT: mechanisms and implications for fibrotic lung disease. Am J Physiol Lung Cell Mol Physiol 2007; 293: L525-L534.

20. Hackett TL, Warner SM, Stefanowicz D, Shaheen F, Pechkovsky DV, Murray LA et al. Induction of epithelial-mesenchymal transition in primary airway epithelial cells from patients with asthma by transforming growth factor-beta1. Am J Respir Crit Care Med 2009; 180: 122-133.

21. Katsuno Y, Lamouille S, Derynck R. TGF-beta signaling and epithelial-mesenchymal transition in cancer progression. Curr Opin Oncol 2013; 25: 76-84.

22. Bartis D, Mise N, Mahida RY, Eickelberg O, Thickett DR. Epithelial-mesenchymal transition in lung development and disease: does it exist and is it important? Thorax 2013; e-pub ahead of print 12 December 2013; doi:10.1136/thoraxjnl-2013-204608.

23. Morbini P, Inghilleri S, Campo I, Oggionni T, Zorzetto M, Luisetti M. Incomplete expression of epithelial-mesenchymal transition markers in idiopathic pulmonary fibrosis. Pathol Res Pract 2011; 207: 559-567.

24. Yamada M, Kuwano K, Maeyama T, Hamada N, Yoshimi M, Nakanishi $Y$ et al. Dual-immunohistochemistry provides little evidence for epithelial-mesenchymal transition in pulmonary fibrosis. Histochem Cell Biol 2008; 129: 453-462.
25. Rock JR, Barkauskas CE, Cronce MJ, Xue Y, Harris JR, Liang J et al. Multiple stromal populations contribute to pulmonary fibrosis without evidence for epithelial to mesenchymal transition. Proc Natl Acad Sci USA 2011; 108: E1475-E1483.

26. Zhu T, Zhang W, Xiao M, Chen H, Jin H. Protective role of andrographolide in bleomycininduced pulmonary fibrosis in mice. Int J Mol Sci 2013; 14: 23581-23596.

27. Yang T, Chen M, Sun T. Simvastatin attenuates TGF-beta1-induced epithelialmesenchymal transition in human alveolar epithelial cells. Cell Physiol Biochem 2013; 31: 863-874.

28. Chen YL, Zhang X, Bai J, Gai L, Ye XL, Zhang $L$ et al. Sorafenib ameliorates bleomycininduced pulmonary fibrosis: potential roles in the inhibition of epithelial-mesenchymal transition and fibroblast activation. Cell Death Dis 2013; 4: e665.

29. Sonnylal S, Xu S, Jones H, Tam A, Sreeram VR, Ponticos M et al. Connective tissue growth factor causes EMT-like cell fate changes in vivo and in vitro. J Cell Sci 2013; 126(Pt 10): 2164-2175.

30. Balli D, Ustiyan V, Zhang Y, Wang IC, Masino AJ, Ren X et al. Foxm1 transcription factor is required for lung fibrosis and epithelial-to-mesenchymal transition. EMBO J 2013; 32: 231-244.

31. Chang CJ, Chao CH, Xia W, Yang JY, Xiong Y, Li CW et al. p53 regulates epithelialmesenchymal transition and stem cell properties through modulating miRNAs. Nat Cell Biol 2011; 13: 317-323.

32. Yang D, Sun Y, Hu L, Zheng H, Ji P, Pecot CV et al. Integrated analyses identify a master microRNA regulatory network for the mesenchymal subtype in serous ovarian cancer. Cancer Cell 2013; 23: 186-199.

33. Thuault S, Valcourt U, Petersen M, Manfioletti G, Heldin CH, Moustakas A. Transforming growth factor-beta employs HMGA2 to elicit epithelial-mesenchymal transition. J Cell Biol 2006; 174: 175-183.

34. Tan EJ, Thuault S, Caja L, Carletti T, Heldin CH, Moustakas A. Regulation of transcription factor Twist expression by the DNA architectural protein high mobility group A2 during epithelial-to-mesenchymal transition. J Biol Chem 2012; 287: 7134-7145.

35. Thuault S, Tan EJ, Peinado H, Cano A, Heldin CH, Moustakas A. HMGA2 and Smads co-regulate SNAIL1 expression during induction of epithelial-to-mesenchymal transition. J Biol Chem 2008; 283: 33437-33446.

36. Yang S, Banerjee S, de Freitas A, Sanders YY, Ding Q, Matalon S et al. Participation of miR-200 in pulmonary fibrosis. Am J Pathol 2012; 180: 484-493.

37. Fu X, Jin L, Wang X, Luo A, Hu J, Zheng X et al. MicroRNA-26a targets ten eleven translocation enzymes and is regulated during pancreatic cell differentiation. Proc Natl Acad Sci USA 2013; 110: 17892-17897.

38. Icli B, Wara AK, Moslehi J, Sun X, Plovie E, Cahill M et al. MicroRNA-26a regulates pathological and physiological angiogenesis by targeting BMP/SMAD1 signaling. Circ Res 2013; 113: 1231-1241.

39. Luo X, Pan Z, Shan H, Xiao J, Sun X, Wang N et al. MicroRNA-26 governs profibrillatory inward-rectifier potassium current changes in atrial fibrillation. J Clin Invest 2013; 123: 1939-1951.

40. Wei C, Kim IK, Kumar S, Jayasinghe S, Hong N, Castoldi G et al. NF-kappaB mediated miR-26a regulation in cardiac fibrosis. J Cell Physiol 2013; 228: 1433-1442.

41. Harada M, Luo X, Qi XY, Tadevosyan A, Maguy A, Ordog B et al. Transient receptor potential canonical-3 channel-dependent fibroblast regulation in atrial fibrillation. Circulation 2012; 126: 2051-2064.

42. Huleihel L, Ben-Yehudah A, Milosevic J, Yu G, Pandit K, Sakamoto K et al. Let-7d microRNA affects mesenchymal phenotypic properties of lung fibroblasts. Am J Physiol Lung Cell Mol Physiol 2014; 306: L534-L542.

43. Yang IV, Coldren CD, Leach SM, Seibold MA, Murphy E, Lin J et al. Expression of cilium-associated genes defines novel molecular subtypes of idiopathic pulmonary fibrosis. Thorax 2013; 68: 1114-1121.

44. Yang B, Lin H, Xiao J, Lu Y, Luo X, Li B et al. The muscle-specific microRNA miR-1 regulates cardiac arrhythmogenic potential by targeting GJA1 and KCNJ2. Nat Med 2007; 13: 486-491.

45. Liang H, Zhang C, Ban T, Liu Y, Mei L, Piao X et al. A novel reciprocal loop between microRNA-21 and TGFbetaRIII is involved in cardiac fibrosis. Int J Biochem Cell Biol 2012; 44: 2152-2160.

(i) () $\odot$ Cell Death and Disease is an open-access journal published by Nature Publishing Group. This work is licensed under a Creative Commons Attribution-NonCommercialNoDerivs 3.0 Unported License. The images or other third party material in this article are included in the article's Creative Commons license, unless indicated otherwise in the credit line; if the material is not included under the Creative Commons license, users will need to obtain permission from the license holder to reproduce the material. To view a copy of this license, visit http://creativecommons.org/ licenses/by-nc-nd/3.0/ 